\title{
Comparison of Federated and Centralized Kalman Filters with Fault Detection Considerations
}

\author{
Paul J. Lawrence, Jr., CAPT, USAF \\ Michael P. Berarducci \\ Air Force Wright Laboratory, WPAFB
}

\begin{abstract}
This paper examines the results obtained by simulating an aircraft navigation system with a partial complement of a typical avionics sensor array using two different techniques of estimation processes: the conventional Kalman and the federated filter architectures. Areas of interest include error state estimation accuracy, residual behavior under induced sensor failure conditions, and potential for failure detection and isolation. Several simulations were accomplished for each filter design and the results were compared in order to verify the validity of the recently developed federated filter architecture. Comparison of the error state estimation accuracies of the two filter designs revealed excellent overall performances for both. The identification of failures showed a definite advantage in the federated filter design. Having sensordedicated local filters allowed for easy sensor failure identification for the federated filter, while the centralized filter design suffered from navigation solution corruption. Once established as a valuable estimation technique, the federated filter will add significantly to the viable alternatives when choosing a filter architecture for avionics modifications or implementations.
\end{abstract}

\section{INTRODUCTION}

The objective of this paper is to provide the results from a comparison of the overall estimation performances between the federated filter design and the well-established Centralized Kalman Filter (CKF) design, along with a comparison of their general respective Failure Detection and Isolation (FDI) capabilities.

The centralized Kalman filter is the most common filter design implemented in the integrated navigation systems of United States Air Force aircraft today. The CKF receives all available measurements and combines all the information contained in those measurements to obtain an optimal navigation solution. For simple, well-modeled linear systems, the CKF is unquestionably the optimal estimator [1]. When considering tradeoffs of data flow, algorithmic requirements, and processing speed versus optimality, fault tolerance, estimation in a multi-sensor environment is often best treated as a distributed estimation problem [1]. The Distributed Kalman Filter (DKF) architectures employ a bank of local Kalman filters dedicated to the sensors which provide measurement information to the system [1]. A master filter combines the estimates from the bank of filters to obtain a typically suboptimal navigation solution. This poses less of a computational burden per filter than a centralized filter implementation. Although these estimates are typically slightly suboptimal, the distributed filter offers improvements over the centralized fault detection and isolation schemes. $[1,2,3,4]$

The advantage of the federated filter architecture is obtained through the sharing of the estimation information by the sensor-dedicated local filters. The recombination of this shared information in the master filter significantly improves the quality of the error state estimates over previous distributed filter designs [1,2,4]. Figure 1 depicts the common structure of a federated filter architecture application to a multi-sensor navigation system.

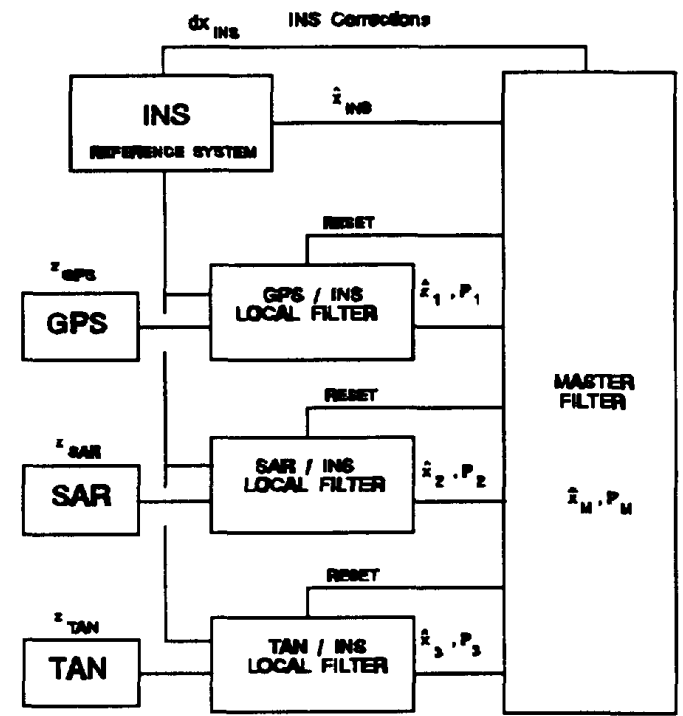

Figure 1 Federated Filter Application to a Multi-

Sensor Navigation System

From the block diagram in Figure 1, the master filter provides the possible reset information to the sensor-dedicated local filters and the correction information to the Inertial Navigation System (INS). The master filter algorithm forms the basis for whether or not the federated filter output is globally optimal $[1,2,3,4,5]$. The local filters receive the measurements directly from the sensors, and then provide the error state information to the master filter for recombination. The INS serves as the reference system 
for the local filters and the master filter. While the Global Positioning System (GPS), Synthetic Aperture Radar (SAR), and Terrain Aided Navigation (TAN) systems are shown, the federated architecture can facilitate more sensor-dedicated filters than this.

The typical distributed filter designs have been characterized as suffering from the cascading effect, which is described briefly by filter-driven-filter instabilities [2]. The federated filter design was deemed the most auspicious choice for implementation of the possible distributed filter designs because it is atypical and does not suffer from those instability difficulties [1]. A means of evaluating this relatively new design was necessary to justify its use in future navigation systems. Integrity Systems Inc. was contracted to provide simulation software for efficiently evaluating distributed Kalman filter architectures. DKFSIM [6] has been presented to Wright Laboratory, Avionics Directorate, Wright-Patterson AFB, OH as the intended simulation tool. Wright Laboratory, Navigation and Information Transmission Branch is the Office of Primary Responsibility (OPR) for the DKFSIM simulation software and the sponsor for this effort. [7]

The federated filter feedback mode selected determines the relative amount of information fed back to the local filters from the master filter. The full and partial fusionreset modes involve feedback of all or part of the master filter fused navigation solution. The zero-reset mode involves no feedback from the master filter, but the local filters retain none of the local information. The no-reset mode involves no feedback from the master filter, and each local filter retains its own unique portion of the total system information. $[2,4,8]$ This paper concentrates on a federated filter in the no-reset mode $[2,4,8]$ with three local filters operating independently on four different sets of sensor output data. The reference system is the Inertial Navigation System (INS), and the external sensor sources are the Global Positioning System (GPS), the Synthetic Aperture Radar (SAR), and the Terrain Aided Navigation (TAN) $[2,3,4,8]$. SARPVU represents precision velocity update SAR measurements, and SAREO represents electrooptic type landmark imaging SAR measurements. A CKF with identical sensor model representations as is used for the federated filter is selected for this comparison.

In order to verify the quality of the federated filter estimation accuracy, it is prudent to compare the federated filter error state performance to that of the centralized filter. An error state accuracy comparison between that of the federated filter and of the centralized filter should indicate, under normal operating conditions, that the centralized filter cannot be out-performed by the federated filter. Error state estimation accuracy can be determined by the state history because it provides a measure of how accurate the filter's estimates are, compared to the real world model. The error state filter formulation used in
DKFSIM estimates the errors in the navigation and attitude information using the difference between the INS and external source information. The dynamics upon which the filter is based is the set of inertial system error propagation equations, which are well developed, well behaved, low frequency, and very adequately represented as linear. Because the filter is based on low frequency dynamics, its sample rate can be much lower than that of a direct filter. For these reasons, the error state space formulation is used in essentially all terrestrial aided inertial navigation systems. [9]

The Failure Detection, Isolation, and Reconfiguration (FDIR) capabilities of the federated filter represent the area where the largest disparity is expected to occur in the performances of the two designs, with substantial advantage being given to the federated filter design. The fault detection aspects of this effort are centered around monitoring the residual outputs provided by each of the independent local filters and that of the centralized filter. The residual sequence is theoretically a white Gaussian sequence of zero-mean with covariance being a function of the observation matrix $\mathbf{H}\left(t_{i}\right)$, the error state covariance $P\left(t_{i}{ }^{-}\right)$, and the measurement process noise $\mathbf{R}\left(\mathbf{t}_{\mathrm{i}}\right)$ :

$$
B\left(x\left(t_{1}\right) x^{T}\left(t_{1}\right)\right)=B\left(t_{1}\right) P\left(t_{1}\right) H^{T}\left(t_{1}\right)+R\left(t_{1}\right)
$$

During operation of the filter, the actual residual sequence can be monitored and compared to this description. If the description appears to be violated consistently, then one can deduce that something has occurred to invalidate the model within the filter. Otherwise, if the violation occurs in only one component of a vector residual process, then it can be assumed that the measuring device generating that particular residual component is the source of the difficulty. [9] Thus, monitoring the residuals of the centralized filter and those at the local filter level in the federated design has the potential to provide accurate fault detection information. The federated filter also provides the capability to monitor local filter fusion residuals [4]. Table 1 lists the residuals used according to the measurements available from the specific sensors.

The filter comparison required a two-stage effort. First, a centralized filter analysis was performed. This included a ten-run Monte Carlo ensemble average of all available error state outputs to facilitate the initial phase of the comparison. Next, the one-run baseline residual output plots which represent ordinary magnitude residuals under normal operating conditions for the next phase of the comparison were obtained. Finally, a small magnitude constant bias failure in the measurements was introduced.

The second stage involved a federated filter analysis with the exact same truth and filter model states as was used for 
Table 1 Residual Listing by Sensor for Both CKF and DKF Designs

\begin{tabular}{|c|c|}
\hline \multicolumn{2}{|c|}{ GPS - 4 Satellite Operation } \\
\hline \multicolumn{2}{|c|}{ Satellites \#1, *2, *3, \#4 } \\
\hline \multicolumn{2}{|c|}{ Pseudorange } \\
\hline \multicolumn{2}{|c|}{ Pseudorange-rate } \\
\hline \multicolumn{2}{|c|}{8 Total GPS Realduals } \\
\hline \multicolumn{2}{|l|}{ SAR } \\
\hline 3 SARPVU & 4 SAREO \\
\hline X-Velocity & Range \\
\hline Y.Velocity & Range-rate \\
\hline Z-Velocity & Elevation \\
\hline & Aximuth \\
\hline \multicolumn{2}{|c|}{7 Total SAR Residuals } \\
\hline \multicolumn{2}{|l|}{ TAN } \\
\hline \multicolumn{2}{|c|}{ Ground clearance } \\
\hline 1 Total TAN & \\
\hline
\end{tabular}

the centralized filter. The ten-run error state estimation performances of the two filter designs were then compared. The baseline residual output plots from the centralized filter and the federated local filters were compared to ensure both filters were operating comparably. Identical relatively small magnitude failures in the sensor measurements for the federated filter were simulated to allow a direct comparison with the centralized filter.

\section{The Federated Kalman Filter Architecture}

Dr. Neal Carlson developed the federated filter architecture as a particular type of distributed filter $[2,3,4]$. The foundation for success of this design is the principle of conservation of information $[2,3,4,8]$. The basic idea of information sharing is to divide the global system information among the several component filters and then perform local processing before recombining the updated local information into a new global sum within the master filter. By recombination we mean a weighted least squares average involving the local estimates and covariances but not involving any cross-covariances. The precise definition of the principle is the "total system information can remain constant or decrease, but never increase, due to sharing". [4]

Without adhering to this information sharing principle, the distributed filter design is subject to poor accuracy and even divergence [2]. This principle allows each local filter estimate to be treated independently by the master filter data fusion process. The master filter simply fuses the weighted state estimates and covariances together to form the globally optimal solution. $[2,3,4]$

The local filters receive sensor measurements and reference system information from the INS. The master filter provides the INS corrections, while combining the information provided by the filters into a globally optimal or suboptimal navigation solution.

For the no-reset mode, the master filter retains none of the fused information, while the local filters collectively retain all of the local information. This no-reset design is highly fault tolerant and, therefore, provides excellent performance for FDI because the local filters operate independently of each other. [2,4] During the propagation cycles, each of the local filters multiplies its common process noise variances by the information sharing fractions in order to share the whole process noise information among them. During measurement update cycles, the local filters perform normal processing of the data from their independent sensors.

\section{FOUNDATIONS FOR COMPARISON}

The truth models are composed of original error states and those states denoted as wide-band noises which are to be incorporated via state augmentation of the original system description [10]. The filter models are composed of all the error state performance histories available for storage in the output data files. Both the truth and filter models used an error state formulation of the INS in EarthCentered-Earth-Fixed (ECEF) coordinates.

\section{The Truth Model State Description}

For the medium accuracy strapdown INS, there are a total of 42 time-correlated truth model states, plus 3 accelerometer and 3 gyro wide-band noise sources.

The GPS truth model assumes four-satellite operation. There are 29 time-correlated GPS truth model states, plus 8 wide-band noise sources. The GPS truth model includes one channel random wide-band phase noise and one channel random frequency wide-band noise per satellite.

The total SAR system utilizes two subsystems for normal operations. There are 20 time-correlated SARPVU and SAREO truth model states, plus 7 wide-band noise sources. The SAR truth model includes one random measurement noise for each of the 7 SAR measurements provided.

There are a total of 33 time-correlated TAN truth model states. The TAN truth model includes random measurement noise on the ground clearance measurement.

The final form of the CKF truth model results in combining the time-correlated and wide-band noise states for a total of 146 truth model error sources. The final form of the DKF truth models depends on the filter designation. Local filter \#1 utilizes only the INS and GPS truth model 
error sources for a total of 85 . Local filter \#2 utilizes only the INS and SAR system truth model error sources for a total of 75 . Local filter \#3 utilizes only the INS and TAN truth model error sources for a total of 82 . The master filter in the no-reset mode utilizes only the INS truth model error sources for a total of 48 .

\section{The Filter Model State Description}

The filter models provided for the CKF and DKF designs are reduced-order implementations of the truth models for each system as appropriate.

The model for the INS includes 16 total filter states. The GPS model includes 5 filter model states (4 satellite/clock range biases and 1 clock frequency bias). The SAR filter models include 3 states for the SARPVU system and 4 states for the SAREO system. Lastly, the TAN model consists of only one filter model state.

The DKF design applies each system's filter state model according to its specified dedicated local filter. Local filter \#1 provides the INS and GPS system filter model states (21). Local filter \#2 provides the INS and SAR system filter model states (23). Local filter \#3 provides the INS and TAN system filter model states (17). The master filter of the DKF design encompasses only those states of the INS filter model (16), while the CKF design encompasses all of the system filter model states (29).

\section{RESULTS AND OBSERVATIONS}

A typical tactical fighter flight profile was used for the analysis and includes ingress to a low level terrain following and terrain avoidance segment, a SAR target acquisition pop-up maneuver, a target bombing run, and egress from the low level portion [1]. The desired simulation data required a series of ten-run and single-run Monte Carlo analyses, accomplished with a flight duration from 0 to 7200 seconds.

\section{The Centralized Versus Federated Filter}

A general comparison can be made with respect to filter performance by comparing the like filter error state performance plots for each filter implementation. For both the centralized and federated filters, the first 16 states correspond to estimates of the errors in the INS and can be compared directly in relative magnitudes of error and their associated one-sigma bounds. The last 13 states likewise correspond to the GPS, SAR, and TAN filter error state performances. The centralized filter provides these state performance outputs based on single filter operations. The federated filter design has the INS error state performances output from the master filter, the GPS error state performances output from local filter \#1, the SAR error state performances output from local filter \#2, and the TAN error state performance output from local filter \#3.

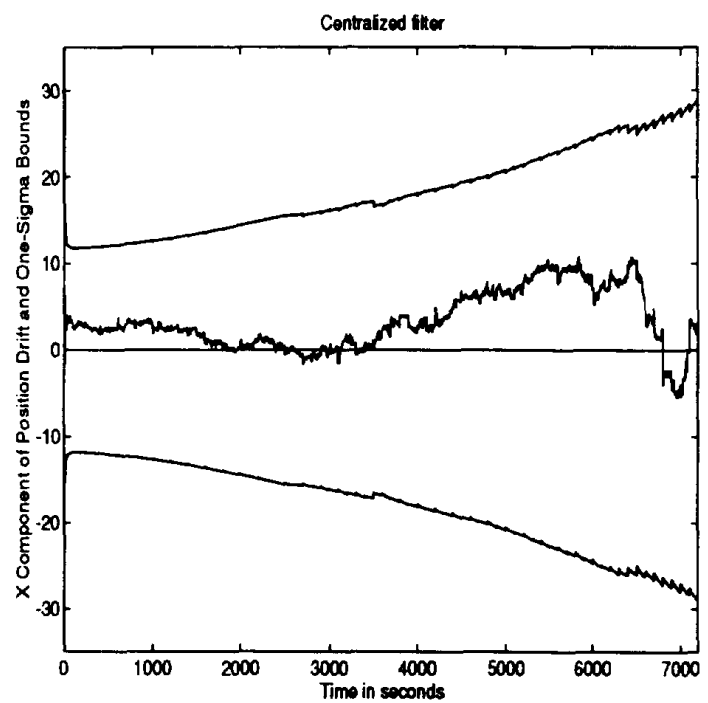

Figure 2 Centralized Kalman Filter, Estimation Error and One-Sigma Bounds for X Component of Position Drift

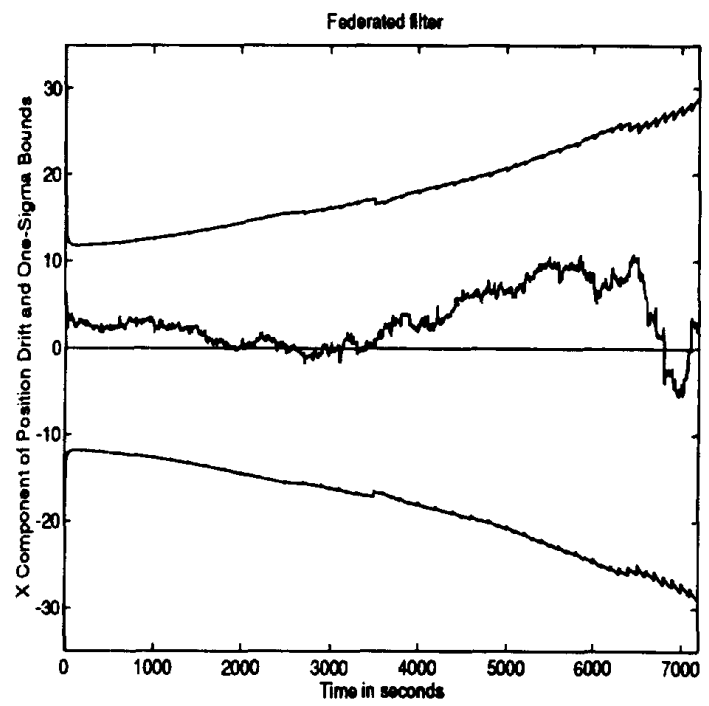

Figure 3 Federated Filter, Estimation Error and OneSigma Bounds for $\mathrm{X}$ Component of Position Drift

The plots of the error state performances indicate how well the filter estimates compare to the magnitudes of the true drifts of the respective states. As seen by the ten-run ensemble averages in Figures 2 and 3, the estimation performances of the two different filter designs are nearly 
identical. These plots of the estimation errors in the $\mathrm{X}$ component of position drift are the only examples provided because all of the INS (in ECEF equatorial coordinates) error state performance plots were very close in comparison. The plots of the estimation errors in the GPS, SAR, and TAN sensor states are also very close with the exception of some divergence in the one-sigma bound values from the federated filter design. This is expected due to the recombination occurring in the master filter.

\section{Fault Detection and Isolation}

Residual tolerance values are selectable for filter residual rejection thresholds from within the DKFSIM input data file. These tolerance values are set so the filter will compare the magnitude of the newly available residual against that of the desired acceptable magnitude. If the residual falls outside of this range of tolerance, the filter will not use this most recent measurement for an update cycle. The filter will propagate the estimate again without an update for this particular cycle. For effective filter fault tolerance, these tolerance values are set low, about 3 to 5 , representing multiples of the one-sigma values, so that the filter does not incorporate the bad information.

The GPS provides a set of eight measurements every five seconds. The four satellite operation requires a pseudorange and a pseudorange-rate residual for each satellite. The SARPVU and SAREO systems provide seven measurements every 100 seconds. The TAN sensor provides one measurement every ten seconds.

The next step for obtaining fault detection evaluations, was to implement the failures by directly corrupting the measurements prior to the calculation of the residuals.

Soft failures were not induced in all the measurements for all the sensors. It was decided that a constant bias on the second GPS measurement, the pseudorange-rate measurement for satellite \#1, and on the TAN measurement would represent a soft constant bias failure quite well for this study. No corresponding constant bias failure was applied to the satellite \#1 pseudorange measurement. The effective time of GPS bias is from 1000 seconds to 3500 seconds and was done by adding a constant value of 0.50 $\mathrm{ft} / \mathrm{sec}$ to the incoming pseudorange-rate measurement. This was deemed a sufficient duration and magnitude for failure and detection. The effective time of the TAN bias is from 4500 seconds to 6500 seconds and was done by adding a constant value of $150 \mathrm{ft}$ to the incoming ground clearance measurement. This provided sufficient time for filter recovery before inducing the TAN failure and allowed for the proper detection magnitude. The TAN and GPS soft failure levels were chosen so that they would not be rejected by a 3 or 5 sigma residual screening test in the filter.

The residual behavior of the pseudorange of satellite \#1 for the federated and centralized filters reflected the greatest impact. Both sets of residuals were alike and

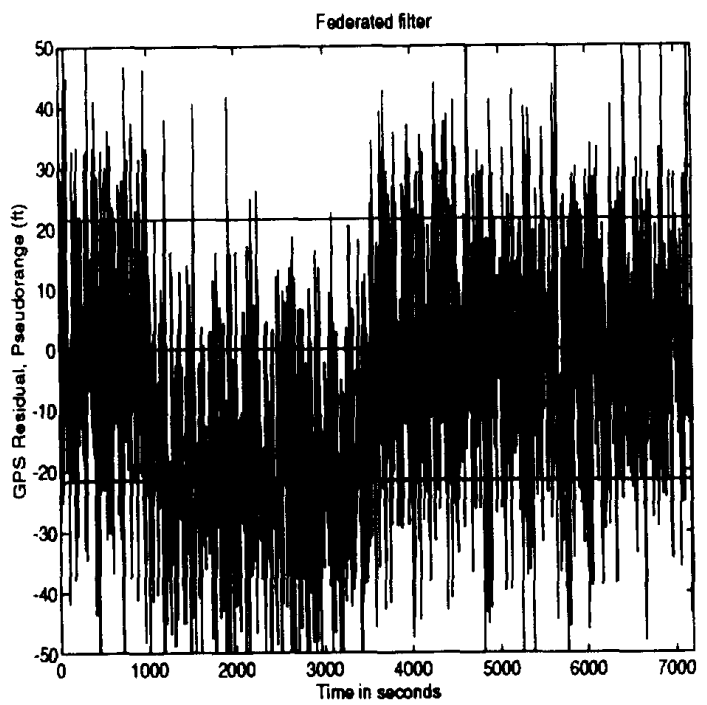

Figure 4 Federated Filter, GPS Satellite \#1 Pseudorange Residual and One-Sigma Bound, Constant Bias

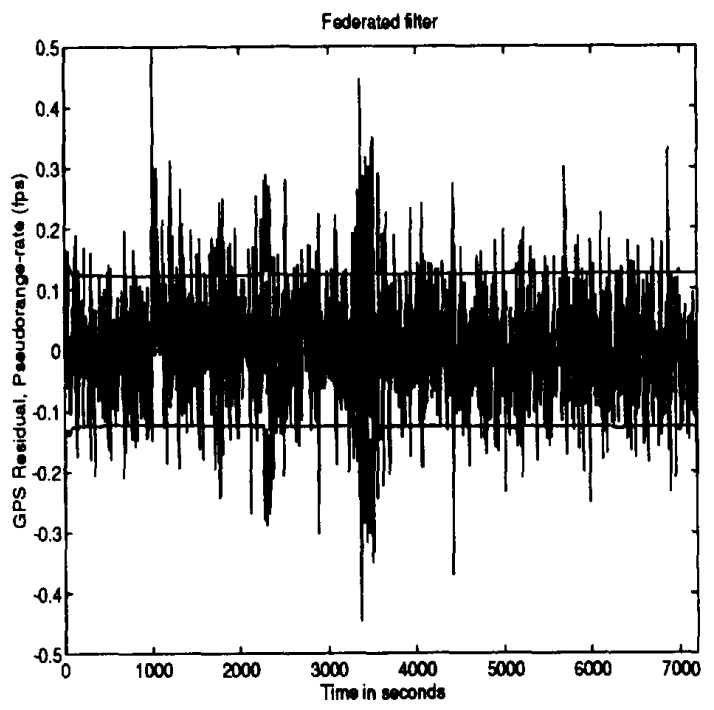

Figure 5 Federated Filter, GPS Satellite \#1

Pseudorange-rate Residual and One-Sigma Bound,

Constant Bias

showed a bias of about 20 feet, see Figure 4. This occurs because, when viewing the pseudorange-rate residual of satellite $\# 1$, the filter has an initial transient due to the constant bias addition at 1000 seconds, and then settles 


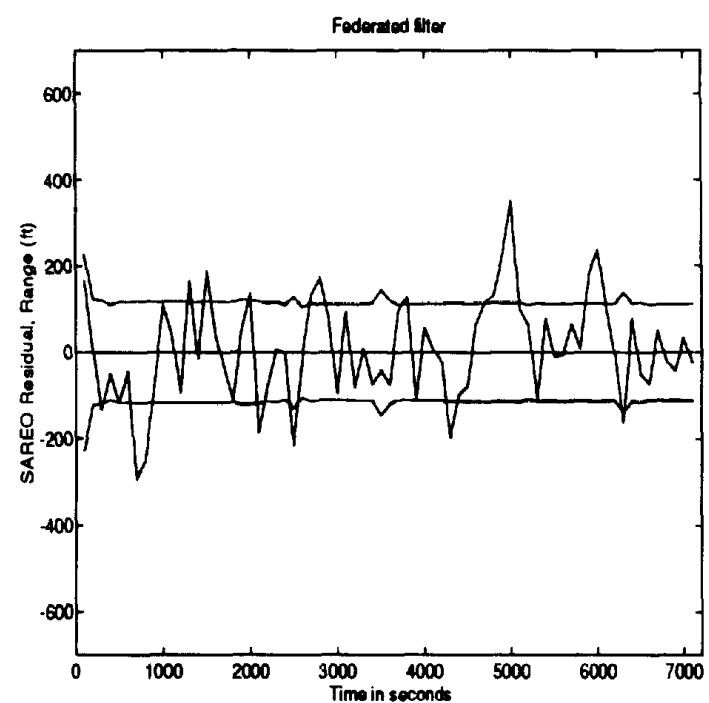

Figure 6 Federated Filter SAREO Range Residual and One-Sigma Bounds, GPS and TAN Constant Bias

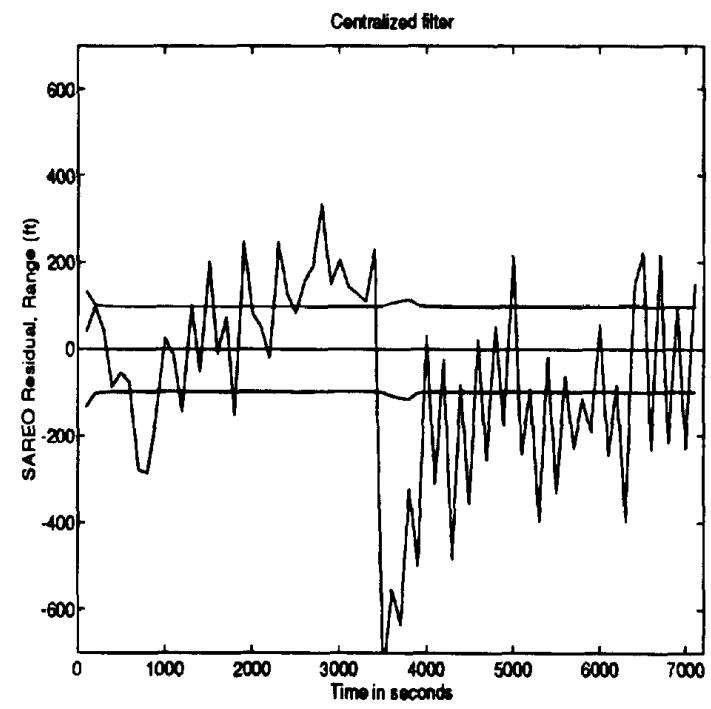

Figure 7 Centralized Filter SAREO Range Residual and One-Sigma Bounds, GPS and TAN Constant Bias

back into normal operations accepting the biased residual as accurate, see Figure 5. Consequently, the pseudorange calculations are impacted by a $0.5 \mathrm{ft} / \mathrm{sec}$ error and the filter is continuously mistaken when calculating the pseudorange. Another transient is experienced when the constant bias is removed, and the filter again settles into normal operations accepting these values as accurate. The filters do not re- ject the biased residuals, but instead use them to update their respective navigation solutions because the fault is less than the residual test failure threshold. The one-sigma bounds for the offset portion in the pseudorange residuals of satellite \#1 for both filters do not increase appropriately because the filters are unaware of the failure and continue on without compensation. Lastly, even though the TAN measurement failed from 4500 to 6500 seconds, the dominance of the GPS accuracy did not allow this failure to impact the GPS residuals even in the centralized filter design. Although they are not presented here, the other GPS pseudorange-rate residuals are affected and show transient behavior because the filter expectation of the residuals is calculated after the most recent update cycle from the one-by-one scalar measurement processing algorithm used in the software.

The real benefit of this comparison is seen when comparing the SAR and TAN residuals of the two filter designs. The federated filter SAR residuals are completely unaffected by the GPS or TAN failures as expected. This is because the SAR dedicated local filter does not have access to the bad measurement information from the GPS or TAN. The SAREO range residuals for the two filter designs showed the most dramatic difference in residual response. Figures 6 and 7 show the SAREO range residuals for the federated and centralized filters, respectively. The centralized filter SAREO residuals are all adversely affected at the time the simulated GPS failure is finally removed. However, the SARPVU residuals of velocity drift are relatively unaffected. Drift indications in the SAREO range residuals do occur before the GPS failure is removed. This indicates a disagreement in the centralized filter between the SAREO and GPS. The additional SAREO range residual errors seen later in the mission hint at an adverse influence from the error induced in the TAN system. There is no actual failure in the SAR system, yet a SAR fault is falsely indicated by the centralized filter and can force the sensor to be taken out of the navigation solution.

The federated filter TAN ground clearance residual plot in Figure 8 shows the residual spikes at 4500 and 6500 seconds and indicates the TAN bias failure.

The centralized filter TAN ground clearance residual in Figure 9 shows the effects of the GPS failure initially and it also shows the relative effects of the SAR residual impact due to the GPS failure.

Because the GPS sensor is the most dominant in accuracy, it would be difficult to detect an impact in the centralized filter's GPS residuals due to any other, lessaccurate sensor failure. However, the no-reset mode of the federated filter, by design, does not allow comption of the other local filter navigation solutions when a particular sensor is failed, regardless of the sensor's accuracy. 


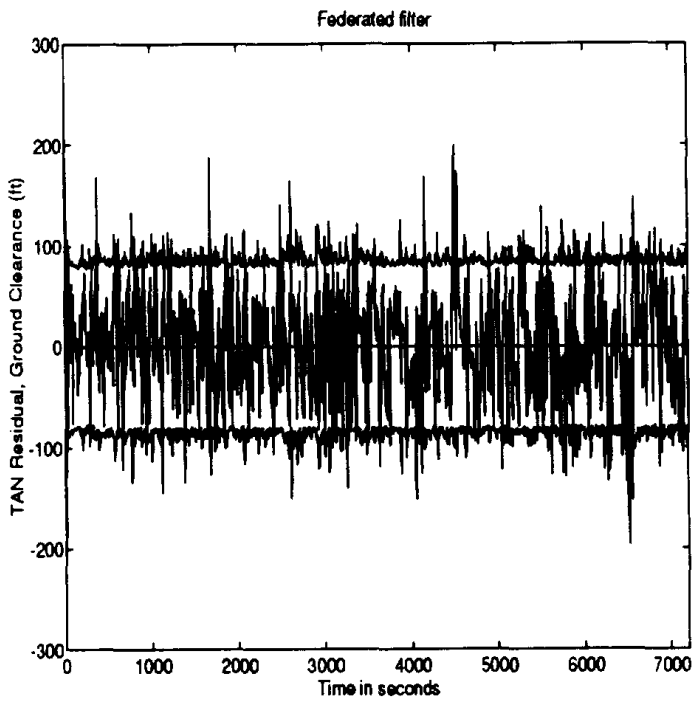

Figure 8 Federated Filter TAN Ground Clearance Residual and One-Sigma Bounds, GPS and TAN Constant Bias

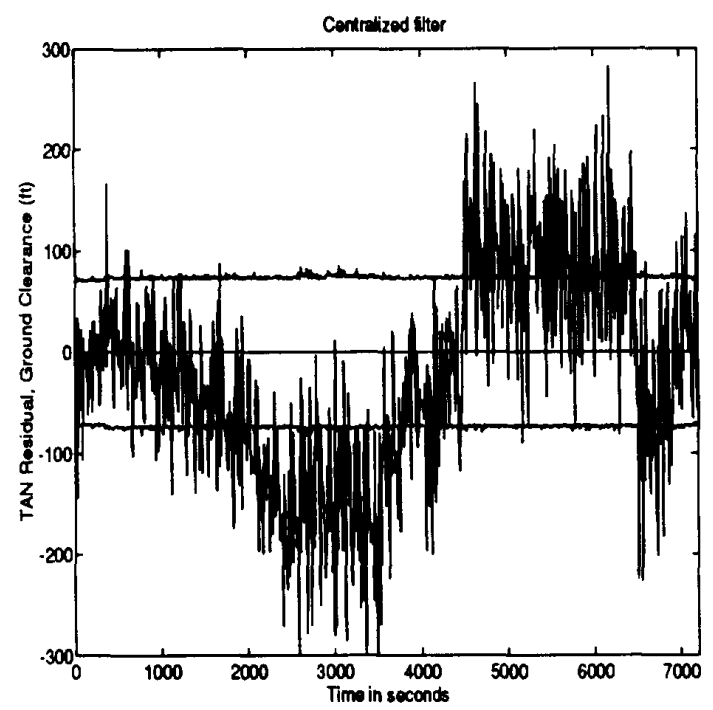

Figure 9 Centralized Filter TAN Ground Clearance Residual and One-Sigma Bounds, GPS and TAN Constant Bias

\section{CONCLUSIONS}

The error-state estimation performance comparison of the centralized and federated filter architectures establishes a reasonable basis for considering the federated filter design as a viable alternative to centralized filtering for future filtering implementations. The two filter designs performed with very nearly the same accuracy when estimating the 29 error states using the ten-run Monte Carlo analysis.

Additional testing under simulated sensor and INS failure conditions would allow an extended comparison of the two filters' performances. Filter stability factors could prove to be an additional deciding factor under certain highdynamic, failed-sensor circumstances. Improved modelling of the sensor systems would allow enhanced simulations involving higher flight dynamics.

\section{Fault Detection and Isolation}

The performances of the two filter designs under failedsensor conditions revealed their respective fault tolerance capabilities. The obvious choice for fault tolerance would have to be the federated filter design using the no-reset mode because the sensor-dedicated filters' measurements and navigation solutions are kept independent.

The centralized filter suffers from the lack of accurate sensor fault identification when a failure occurs. Note that the exception is the hard failure which occurs and is detected immediately, and subsequently the filter rejects that bad information. If the detection is not immediate and the failure information is incorporated into the navigation solution, missed detections and false alarms appear inevitable. Thus, a perfectly good sensor may be removed from the navigation solution. The GPS is so dominant that the filter would have more confidence in its measurement information than that of the SAR or TAN under these circumstances. This is the reason for the GPS residuals not reflecting any impact from the TAN failures.

The federated filter allows for a more efficient voting scheme because the residuals from the local filters are not affected by the other sensor's bad information. This is limited to the level of accuracy of each of the respective sensors. It would take a great deal of error on the part of the GPS before the TAN and SAR could vote that filter out of the master filter navigation solution.

Additionally, failure modelling would allow more complicated failure modes in the sensor operations. Perhaps, for the GPS system, allowing for availability of less than four satellites would prove fruitful for further study.

Further, INS failure modes could easily be implemented in source code. Gradual failures of the INS appear to be a topic of great concern. The failure simulations in the SAR and TAN systems can be relatively de-emphasized in 
both filter implementations because the GPS obviously dominates the estimation processes due to its greater accuracy. The SAR and TAN systems proved most useful when considering the impact of GPS failures on other systems and the aspects of filtering.

Perhaps, one of the most important aspects of fault detection is the application of the detection algorithm. If the algorithm was included in the simulation such that it could provide detection information to the output data files, then failure analysis, and then possibly system reconfiguration could be performed on line while the simulation is being performed. This might also include some Multiple Model Adaptive Estimation (MMAE) considerations [11]. Thus, enhancing the possibilities for system reconfiguration testing under failed subsystem conditions.

\section{Summary}

The potential for follow-on study is great in this area. The federated filter is an excellent implementation of filtering theory. Comparisons of the federated design to the centralized design will likely be carried on into the twenty-first century. Hopefully, any advancements in filtering theory which enable engineers to simplify the task of obtaining a navigation solution under normal or failed operating conditions will be utilized to their full advantage. This study definitely shows that the federated filter is worthy of serious consideration for integrated navigation.

\section{REFERENCES}

1. Denaro, Loomis, and Geier. Common Kalman Filter Development. Document No. WRDC-TR-89-1152. Wright-Patterson AFB, OH, Feb 1990.

2. Carison, Neal. "Federated Filter for Fault-Tolerant Integrated Navigation Systems". IEEE PLANS, Position Location And Navigation Symposium. 1988:

1-10 (Dec 88).

3. Carlson, Neal. "Federated Square Root Filter for Decentralized Parallel Processes". IEEE Transactions on Aerospace and Electronic Systems, 26: No. 3, 517-525 (Apr 1989).

4. Carlson, Neal and Berarducci, Michael. "Federated Kalman Filter Simulation Results". Institute of Navigation (ION) 49th Annual Meeting, Cambridge, MA: (Jun 1993).

5. Smith, M.S. and G.S. Ladde. "Processing of Prefiltered GPS Data", IEEE Transactions on Aerospace and Electronic Systems, 25: No. 5, 711-727 (Sep 1989).

6. DKFSIM 1.1 User's Manual. Document No. TM-89-005. Integrity Systems, Inc., Winchester, MA, Dec 1989.

7. Lawrence, Paul J., Jr. Comparison of a Distributed Kalman Filter Versus a Centralized Kalman Filter With Fault Detection Considerations. MS Thesis Document No.
AFIT/GE/ENG/93S-06, School of Engineering, Air Force Institute of Technology, Wright-Patterson AFB, OH, Jul 1993.

8. Gao, Y. and others. "Experience With the Application of Federated Filter Design to Kinematic GPS Positioning". IEEE PLANS, Position Location and Navigation Symposium, 1992: 314-320 (Mar 1992).

9. Maybeck, Peter S. Stochastic Models, Estimation, and Control, Volume 1. San Diego, CA: Academic Press, 1979.

10. Carlson, Neal. Technical Operating Report \#3: Distributed Kalman Filter Mission Performance Simulation Plan. Document No. TM-89-001-2. Integrity Systems, Inc., Winchester, MA, May 1989.

11. Martin, Daniel and Carlson, Neal. Distributed Model Adaptive Estimation, Phase I, Final Report. Document No. WL-TR-92-1106, Wright-Patterson AFB, OH, Aug 1991. 